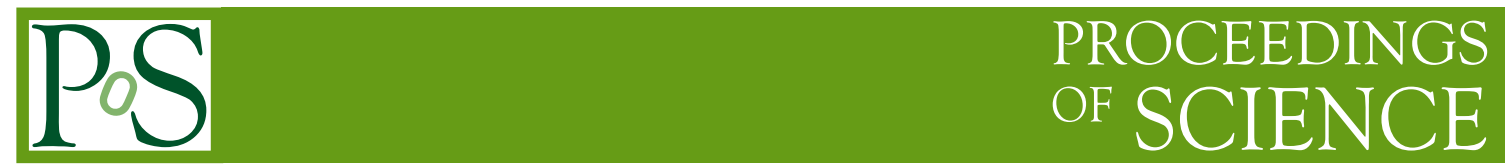

\title{
Magnetic moment of the neutrino
}

\author{
Alexander Studenikin* \\ Department of Theoretical Physics, Faculty of Physics, Lomonosov Moscow State University, \\ Moscow 119991, Russia \\ Joint Institute for Nuclear Research, Dubna 141980, Moscow Region, Russia \\ E-mail: studenikdsrd.sinp.msu.ru
}

We recall the main issues related to the neutrino magnetic moment, and then we summarize the corresponding most important and recently discussed effects that can have phenomenological consequences.

Neutrino Oscillation Workshop (NOW2018)

9 - 16 September, 2018

Rosa Marina (Ostuni, Brindisi, Italy)

${ }^{*}$ Speaker. 
Present status. There is no doubt [1-3] that neutrino electromagnetic properties open a window to new physics. The most general form [四] of a neutrino electromagnetic vertex function $\Lambda_{\mu}^{i j}(q)=\left(\gamma_{\mu}-q_{\mu} q / q^{2}\right)\left[f_{Q}^{i j}\left(q^{2}\right)+f_{A}^{i j}\left(q^{2}\right) q^{2} \gamma_{5}\right]-i \sigma_{\mu \nu} q^{v}\left[f_{M}^{i j}\left(q^{2}\right)+i f_{E}^{i j}\left(q^{2}\right) \gamma_{5}\right]$, where $\Lambda_{\mu}(q)$ and form factors $f_{Q, A, M, E}\left(q^{2}\right)$ are $3 \times 3$ matrices in the space of massive neutrinos, in the case of coupling with a real photon $\left(q^{2}=0\right)$ provides four sets of neutrino electromagnetic characteristics: 1$)$ the dipole magnetic moments $\left.\mu_{i j}=f_{M}^{i j}(0), 2\right)$ the dipole electric moments $\left.\varepsilon_{i j}=f_{E}^{i j}(0), 3\right)$ the millicharges $q_{i j}=f_{Q}^{i j}(0)$ and 4) the anapole moments $a_{i j}=f_{A}^{i j}(0)$. The study of the electromagnetic properties of neutrinos attracts considerable attention.

The most well understood and studied are the dipole magnetic moments. In a minimal extension of the Standard Model the diagonal magnetic moment of a Dirac neutrino is given [䧃] by $\mu_{i i}^{D}=\frac{3 e G_{F} m_{i}}{8 \sqrt{2} \pi^{2}} \approx 3.2 \times 10^{-19}\left(\frac{m_{i}}{1 \mathrm{eV}}\right) \mu_{B}$ ( $\mu_{B}$ is the Bohr magneton). Majorana neutrinos can have only transition (off-diagonal) magnetic moments $\mu_{i \neq j}^{M}$. The most stringent constraints on the effective neutrino magnetic moment are obtained with the reactor antineutrinos: $\mu_{v}<2.9 \times 10^{-11} \mu_{B}$ (GEMMA Collaboration [[]]), less stringent constraints were also obtained by the TEXONO Collaboration [焑]. The study of the solar neutrinos fluxes yield slightly more stringent constraints: $\mu_{v_{e}} \leq 2.8 \times 10^{-11} \mu_{B}$ (Borexino Collaboration [四]).

An astrophysical bound (for both Dirac and Majorana neutrinos) is provided [ [ 8$]$-[एत] by observations of the properties of globular cluster stars: $\left(\sum_{i, j}\left|\mu_{i j}\right|^{2}\right)^{1 / 2} \leq(2.2-2.6) \times 10^{-12} \mu_{B}$. A general and termed model-independent upper bound on the Dirac neutrino magnetic moment, that can be generated by an effective theory beyond a minimal extension of the Standard Model, has been derived in [U]]: $\mu_{v} \leq 10^{-14} \mu_{B}$. The corresponding limit for transition moments of Majorana neutrinos is much weaker [12]].

Future prospects. The foreseen progress in constraining the neutrino magnetic moments is related, first of all, with the expected new results from the GEMMA experiment measurements of the reactor antineutrino cross section on electrons at the Kalinin Power Plant. The new set of data is expected to arrive next year. The electron energy threshold will be as low as $350 \mathrm{eV}$ ( or even lower, up to $\sim 200 \mathrm{eV}$ ). This will provide possibility to test the neutrino magnetic moment on the level of $\mu_{v} \sim 0.9 \times 10^{-12} \mu_{B}$. Note that data on the neutrino scattering obtained by the GEMMA experiment can be also used [[13] for constraining the neutrino millicharge.

For the future progress in studying (or constraining) the neutrino magnetic moments a rather promising claim was made in [44, [5]]. It was shown that even tine values of the Majorana neutrino transition moments would probably be tested in future high-precision experiments with the astrophysical neutrinos. In particular, observations of supernova fluxes in the JUNO experiment (see [16-18] may reveal the effect of collective spin-flavour oscillations due to the Majorana neutrino transition moment $\mu_{v}^{M} \sim 10^{-21} \mu_{B}$.

Recent studies on neutrino magnetic moments. The most interesting and important among the consequences of nonzero neutrino magnetic moments are the neutrino spin procession in the transversal magnetic fields [团], [19-21]. In the presence of matter [22-24] the corresponding spin $v_{f}^{L} \Leftrightarrow v_{f}^{R}$ and spin-flavour $v_{f}^{L} \Leftrightarrow v_{f^{\prime}}^{R}\left(f \neq f^{\prime}\right)$ oscillations can be amplified due to the resonance, the effect that can have important consequences in different astrophysical settings. Recently, a thorough study of neutrinos electromagnetic interactions, accounting in particular for magnetic moments, in the theoretical formulation of low-energy elastic neutrino-electron scattering is pre- 
sented in [25]. In [26] on the bases of the exact solution of the neutrino evolution equation the influence of neutrino magnetic moments on the propagation of ultrahigh-energy cosmic neutrinos in the interstellar space is carried out. Within the developed new approach (see [D6], [R7] and references therein) to description of neutrino flavour, spin and spin-flavor oscillations the corresponding probabilities are calculated accounting for the whole set of possible conversions between four neutrino states $\left(v_{e}^{L}, v_{e}^{R}, v_{\mu}^{L}, v_{\mu}^{R}\right)$. It has been shown, in particular, that in the presence of the transversal magnetic field: 1) the amplitude of the flavour oscillations $v_{e}^{L} \Leftrightarrow v_{\mu}^{L}$ at the vacuum frequency is modulated by the magnetic field frequency $\omega_{B}=\mu_{v} B_{\perp}$, 2) the neutrino spin oscillation $v_{e}^{L} \Leftrightarrow v_{e}^{R}$ probability exhibits the dependence on the mass square difference $\Delta m^{2}$. The discussed interplay of neutrino oscillations in magnetic fields on different frequencies can have important consequences in astrophysical environments, in particular in those peculiar for magnetars [27]. The quantum approach to neutrino spin and spin-flavour oscillations engendered by the matter transversal current or polarization, the effect predicted in [29], has been developed in [[28].

As it has been shown in [30], the most favorable conditions for the spin light of neutrino in matter (that is another possible manifestations of a nonzero neutrino magnetic moment) is provided by ultra-dense matter of neutron stars and ultra-high energy of the radiating neutrino, and these conditions can be met within galaxy clusters.

The author is thankful to Paolo Bernardini, Gianluigi Fogli and Eligio Lisi for the invitation to attend the Neutrino Oscillation Workshop and to all of the organizers for hospitality. This work is supported by the Russian Basic Research Foundation grants No. 16-02-01023 and 17-52-53133.

\section{References}

[1] C. Giunti and A. Studenikin, Neutrino electromagnetic interactions: a window to new physics, Rev. Mod. Phys. 87 (2015) 531.

[2] A. Studenikin, Neutrino magnetic moment: a window to new physics, Nucl. Phys. Proc. Suppl. 188 (2009) 220.

[3] A. Studenikin, Neutrino electromagnetic properties: a window to new physics - II, PoS-EPS-HEP2017 (2017) 137.

[4] K. Fujikawa and R. Shrock, The magnetic moment of a massive neutrino and neutrino spin rotation, Phys. Rev. Lett. 45 (1980) 963.

[5] A. G. Beda et al., The results of search for the neutrino magnetic moment in GEMMA experiment, Adv. High Energy Phys. 2012 (2012) 350150.

[6] H. T. Wong and H. B. Li, Neutrino magnetic moments, Mod. Phys. Lett. A 20 (2005) 1103.

[7] M. Agostini et al. [Borexino Collaboration], Limiting neutrino magnetic moments with Borexino Phase-II solar neutrino data, Phys. Rev. D 96 (2017) 091103.

[8] G. G. Raffelt, New bound on neutrino dipole moments from globular-cluster stars, Phys. Rev. Lett. 64 (1990) 2856.

[9] N. Viaux, et al., Particle-physics constraints from the globular cluster M5: neutrino dipole moments, Astron. Astrophys. 558 (2013) A12.

[10] S. Arceo-Díaz, K.-P. Schröder, K. Zuber and D. Jack, Constraint on the magnetic dipole moment of neutrinos by the tip-RGB luminosity in $\omega$-Centauri, Astropart. Phys. 70 (2015) 1. 
[11] N. F. Bell, V. Cirigliano, M. J. Ramsey-Musolf et al How magnetic is the Dirac neutrino?, Phys. Rev. Lett. 95 (2005) 151802.

[12] N. F. Bell, M. Gorchtein, M. J. Ramsey-Musolf, P. Vogel and P. Wang, Model independent bounds on magnetic moments of Majorana neutrinos, Phys. Lett. B 642 (2006) 377.

[13] A. Studenikin, New bounds on neutrino electric millicharge from limits on neutrino magnetic moment, Europhys. Lett. 107 (2014) 21001.

[14] A. de Gouvea and S. Shalgar, Effect of transition magnetic moments on collective supernova neutrino oscillations, JCAP 1210 (2012) 027.

[15] A. de Gouvea and S. Shalgar, Transition magnetic moments and collective neutrino oscillations: Three-flavor effects and detectability, JCAP 1304 (2013) 018.

[16] F. An et al. [JUNO Collaboration], Neutrino physics with JUNO, J. Phys. G 43 (2016) 030401.

[17] C. Giunti, K. Kouzakov, Y. F. Li, A. Lokhov, A. Studenikin, S. Zhou, Electromagnetic neutrinos in laboratory experiments and astrophysics, Annalen Phys. 528 (2016) 198.

[18] J.S. Lu, Y.-F. Li and S. Zhou, Getting the most from the detection of Galactic supernova neutrinos in future large liquid-scintillator detectors, Phys. Rev. D 94 (2016) 023006.

[19] A. Cisneros, Effect of neutrino magnetic moment on solar neutrino observations, Astrophys. Space Sci. 10 (1971) 87.

[20] J. Schechter and J. W. F. Valle, Majorana neutrinos and magnetic fields, Phys. Rev. D 24 (1981) 1883.

[21] L. Okun, M.Voloshin and M.Vysotsky, Neutrino electrodynamics and possible effects for solar neutrinos, Sov. Phys. JETP 64 (1986) 446.

[22] E. Akhmedov, Resonant amplification of neutrino spin rotation in matter and the solar neutrino problem, Phys. Lett. B 213 (1988) 64.

[23] C.-S.Lim and W. Marciano, Resonant spin-flavor precession of solar and supernova neutrinos, Phys. Rev. D 37 (1988) 1368.

[24] G. Likhachev and A. Studenikin, Neutrino oscillations in the magnetic field of the sun, supernovae, and neutron stars, J. Exp. Theor. Phys. 81 (1995) 419 [Zh. Eksp. Teor. Fiz. 108 (1995) 769].

[25] K. Kouzakov and A. Studenikin, Electromagnetic properties of massive neutrinos in low-energy elastic neutrino-electron scattering, Phys. Rev. D 95 (2017) 055013.

[26] P. Kurashvili, K. A. Kouzakov, L. Chotorlishvili and A. I. Studenikin, Spin-flavor oscillations of ultrahigh-energy cosmic neutrinos in interstellar space: The role of neutrino magnetic moments, Phys. Rev. D 96 (2017) 103017.

[27] A. Popov and A. Studenikin, Neutrino eigenstates and flavour, spin and spin-flavour oscillations in a constant magnetic field, Eur. Phys. J. C 79 (2019) 144, arXiv:1803.05755, arxiv:1902.08197 [hep-ph].

[28] P. Pustoshny and A. Studenikin, Neutrino spin and spin-flavour oscillations in transversal matter currents with standard and non-standard interactions, Phys. Rev. D 98 (2018) 113009. arXiv:1808.00302 [hep-ph].

[29] A. I. Studenikin, Neutrinos in electromagnetic fields and moving media, Phys. Atom. Nucl. 67 (2004) 993 [Yad. Fiz. 67 (2004) 1014].

[30] A. Grigoriev, A. Lokhov, A. Studenikin and A. Ternov, Spin light of neutrino in astrophysical environments, JCAP 1711 (2017) no.11, 024. 